\title{
Editorial
}

\section{p53 stabilization: the importance of nuclear import}

\author{
J-C Marine ${ }^{\star, 1}$ \\ Cell Death and Differentiation (2010) 17, 191-192; doi:10.1038/cdd.2009.183
}

In response to cellular stress, the p53 tumor suppressor protein is stabilized as a result of inactivation of ubiquitinmediated degradation. The $\mathrm{p} 53$ protein rapidly accumulates in the nucleus, where it functions as a transcription factor. Although the regulation of p53 stability has been studied extensively, the subcellular compartment in which p53 degradation takes place in unstressed cells is still a matter of debate. On the one hand, studies using ectopic expression of nuclear localization signal (NLS) and nuclear export signal (NES) mutants of p53 and MDM2, the main E3 ubiquitin ligase of $p 53$, have suggested that relocalization to the cytoplasm is necessary for efficient p53 degradation. On the other hand, when ectopic p53 and Mdm2 are prevented from leaving the nucleus, p53 is efficiently ubiquitylated and degraded. ${ }^{1}$ Moreover, efficient degradation of endogenous p53 in the nucleus has been shown during the recovery period after DNA damage. $^{2}$ It therefore seems that both cytoplasmic and nuclear proteasomes are capable of degrading p53.

In this issue, Marchenko et al..$^{3}$ analyze ubiquitylation and degradation of endogenous p53 in unstressed cells using nuclear/cytoplasmic fractionation. The authors observed that ubiquitylated p53 mainly localizes to the cytoplasm, even under conditions of proteasome inhibition and nuclear export block. In other words, in unstressed cells the predominant pool of p53 is born and dies in the cytoplasm, and is subject to constant rapid turnover (Figure 1). This is because there is more MDM2 in the cytoplasm and therefore its interaction with p53 is higher there. However, as in unstressed cells p53 degradation occurs mainly in the cytoplasm, one could expect it to accumulate in this compartment upon stress - yet p53 accumulates in the nucleus. The answer - as Marchenko et al. show - lies in the regulated p53 import that depends on the ubiquitin modification status of p53. This status differs greatly between unstressed and stressed cells.

NLS and NES motifs facilitate the shuttling of p53 in unstressed cells between the nucleus and the cytoplasm. Although p53 NLSs (main NLS I and two accessory NLSs) were first identified almost 20 years ago, ${ }^{4}$ surprisingly, there is virtually nothing known about the regulation of p53 nuclear import and its role in p53 function.

Nuclear import is a relatively common way of regulating the activity of a transcription factor. For example, in the interferon response pathway, STAT1 nuclear localization is regulated by tyrosine phosphorylation; this leads to dimerization that induces a conformational change revealing an NLS, which mediates nuclear import when bound by importin- $\alpha 5$. $^{5}$ Although ubiquitylation is best known for its role in proteasomal degradation it has also been implicated in subcellular trafficking, including the regulation of nuclear export ${ }^{6}$ and nuclear import, ${ }^{7,8}$ and in mitochondrial targeting. ${ }^{9}$ For instance, the nuclear import of PTEN, mainly a plasmamembrane-associated lipid phosphatase that inhibits PI3KAKT signaling, is positively regulated by ubiquitylation. The importance of this nuclear PTEN pool for PTEN-mediated tumor suppression was discovered via a nuclear-excluded but enzymatically intact germline PTEN mutant in intestinal polyps from a patient with Cowden syndrome. Forced monoubiquitylation of mutant PTEN overcame its defective nuclear import. ${ }^{7}$ Interestingly, it has been speculated that nuclear localization of PTEN might also protect it from cytoplasmic polyubiquitylation and degradation. ${ }^{10}$ By analogy, nuclear import of p53 in response to stress may serve as a rapid mechanism of p53 stabilization by removing it from the cytoplasm, its major site of degradation.

In their paper, Marchenko et al. provided data in support of this model. They showed for the first time that ubiquitylation of p53 negatively regulates its nuclear import. Consequently, the concentration of nuclear p53 is low in unstressed cells (Figure 1). They identified the mechanism of this regulation as being critically linked to p53's NLS I. In unstressed cells, ubiquitylation of lysines 319-321 of NLS I interferes with p53's binding to importin- $\alpha 3$, the p53-specific adaptor of the import machinery. Thus, p53 is trapped in the cytoplasm and is unprotected from local degradation. The small pool of p53 that either undergoes HAUSP-mediated deubiquitylation or escapes NLS I ubiquitylation interacts with the import machinery, supplying a constant but low level of nuclear p53 in unstressed cells (Figure 1).

Marchenko et al. went on to demonstrate that in response to stress, MDM2 and p53 modifications rapidly and dramatically reduce the level of ubiquitylation, leading to the accumulation of non-ubiquitylated p53 in the cytoplasm. This renders NLS I competent for efficient importin- $\alpha 3$ recognition by unmasking the basic charges of NLS I, which they show is critical for importin- $\alpha 3$ recognition. The current model of stress-induced nuclear stabilization rests solely on the notion that tetramerization of nuclear p53 masks its NES, thereby causing export block and trapping p53 in the nucleus. ${ }^{11}$

\footnotetext{
${ }^{1}$ Laboratory For Molecular Cancer Biology, Flanders Interuniversity Institute for Biotechnology, 927 Technologiepark, Ghent 9052, Belgium

${ }^{*}$ Corresponding author: J-C Marine, Laboratory For Molecular Cancer Biology, Flanders Interuniversity Institute for Biotechnology, 927 Technologiepark, Ghent 9052 , Belgium. Tel: + 329331 3640; Fax: + 329331 3516; E-mail: chris.marine@dmbr.ugent.be
} 


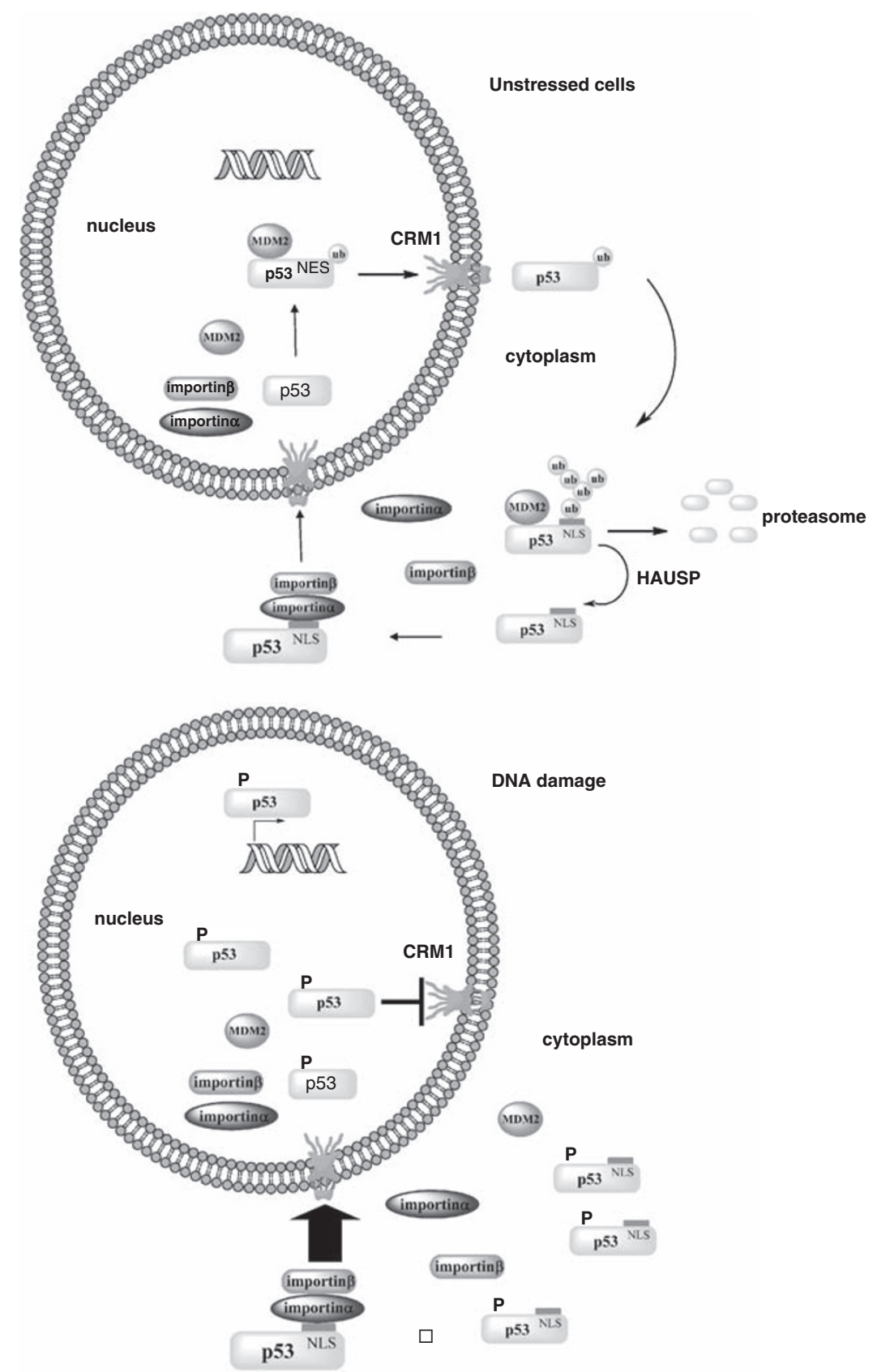

Figure 1 Expanded model of stress-mediated nuclear stabilization of p53 involving a rapid phase of nuclear import and a slower phase of blocked nuclear export

However, Marchenko et al.'s kinetic studies revealed that nuclear accumulation of p53 after stress by far outpaces the kinetics of export block. Conversely, they see a time-dependent interaction between endogenous p53 and importin- $\alpha 3$ shortly after exposure to DNA-damaging agents. Thus, the authors propose an expanded model in which enhanced nuclear import actively contributes to the early phase of nuclear stabilization of p53 in response to stress, an event that is obviously crucial for its transcriptional function. During the later stages of stress response, blocked nuclear export further contributes to its nuclear stabilization (Figure 1).

\section{Conflict of interest}

The authors declare no conflict of interest.

1. Xirodimas DP, Stephen CW, Lane DP. Exp Cell Res 2001; 270: 66-77.

2. Shirangi TR, Zaika A, Moll UM. FASEB J 2002; 16: 420-422.

3. Marchenko ND et al. Cell Death Differ 2009; 17: 255-267.

4. Shaulsky G et al. Mol Cell Biol 1990; 10: 6565-6577.

5. McBride KM et al. EMBO J 2002; 21: 1754-1763.

6. Li M et al. Science 2003; 302: 1972-1975.

7. Trotman LC et al. Cell 2007; 128: 141-156.

8. van der Horst A et al. Nat Cell Biol 2006; 8: 1064-1073.

9. Marchenko ND, Moll UM. Cell Cycle 2007; 6: 1718-1723.

10. Salmena L, Pandolfi PP. Nat Rev Cancer 2007; 7: 409-413.

11. Stommel JM et al. EMBO J 1999; 18: 1660-1672. 